\title{
Tholins as Coloring Agents on Outer Solar System Bodies
}

\author{
Dale P. Cruikshank ${ }^{a}$, Hiroshi Imanaka ${ }^{a, b}$, and Cristina M. Dalle Ore ${ }^{a, b}$ \\ aNASA Ames Research Center, MS 245-6 Moffett Field, CA 94035-1000, USA \\ [Dale.P.Cruikshank@nasa.gov] \\ ${ }^{b}$ SETI Institute, 515 N. Whisman Road, Mountain View, CA 94043, USA
}

\begin{abstract}
The red colors of many solid bodies in outer Solar System may be caused by tholins, which are refractory organic complexes, incorporated in their surface materials. Tholins synthesized in the laboratory are shown to match the colors of these bodies when their optical properties are used in rigorous scattering models. We review recent successes in modeling the spectra of icy outer Solar System bodies with tholins as the coloring agents. New work on the systematic laboratory synthesis and analysis of tholins made by cold plasma discharge in mixtures of gaseous $\mathrm{CH}_{4} / \mathrm{N}_{2}$ shows that the composition of the tholin depends strongly on the pressure in the reaction chamber, and only weakly on the mixing fraction of $\mathrm{CH}_{4}$ relative to $\mathrm{N}_{2}$. In tholins made at high pressure (e.g., $23 \mathrm{hPa}$ ) the abundance of aliphatic hydrocarbons is greater and the abundance of aromatic hydrocarbons is less than in tholins made at low pressure (e.g., $0.13 \mathrm{hPa}$ ). Tholins made at low deposition pressures show a greater abundance of $\mathrm{N}-\mathrm{H}$ bonds.
\end{abstract}

\section{Keywords:}

\section{The Colors of Small Bodies in the Outer Solar System}

Solid bodies in the outer Solar System (OSS) exhibit colors ranging from neutral to very red. Color is defined as the gradient in the spectral reflectance with increasing wavelength, after correction for the color of the illuminating source, the Sun. Objects of low albedo $(0.01-0.15)$ commonly show a red slope to varying degrees and extending somewhat longer than $1 \mu \mathrm{m}$. Icy planetary satellites with higher albedo $(0.2-1.0)$ also have varying degrees of color, but the positive spectral reflectance gradient of these bodies is usually limited to the wavelength region $0.2-0.6 \mu \mathrm{m}$. The Moon is red because of a space weathering phenomenon in which particles in the solar wind sputter and reduce small amounts of iron in the iron-bearing surface minerals. The reduced nano-phase $\mathrm{Fe}$ is deposited on adjacent grains, altering the spectral reflectance and rendering it red in color (Pieters et al. 2000, Hapke 2001). The same space weathering phenomenon occurs on many asteroids in the Main Belt (Hapke 2001). Mars is red because of its surface coating of oxides of iron (e.g., Binder and Cruikshank 1966), and the red and orange colors of Io result from the various polymorphs of sulfur covering its volcanic regions. Both Mars and Io have much higher albedos than the objects we address in this paper. 


\section{Materials that May Impart Red Color}

While a modest degree of red color, such as that of Trojan asteroid 624 Hektor can be explained by the presence of the mineral $\mathrm{Mg}$-rich pyroxene (Cruikshank et al. 2001; Emery and Brown 2004), redder objects require other materials that are not minerals. Gradie and Veverka (1980) introduced the concept of organic solids to explain the red color of the D-type asteroids, suggesting the presence of "...very opaque, very red, polymer-type organic compounds, which are structurally similar to aromatic-type kerogen". They compared the reflectance spectra of the D-type asteroids to mixtures of montmorillonite (clay), magnetite (iron oxide), carbon black, and a coal-tar residue (kerogen) that was insoluble in organic solvents, finding a satisfactory match for both the low albedo and the red color of this specific class of asteroids. Kerogen-like structures are found in carbonaceous meteorites (Kerridge et al. 1987), and the presence of similar structures in interstellar dust grains has also been deduced from infrared spectra of dusty diffuse interstellar clouds (Pendleton and Allamandola 2002).

Tholins, which are the refractory residues from the irradiation of gases and ices containing hydrocarbons, have color properties that make them reasonable candidates for comparison to the spectra of Solar System bodies. Furthermore, they are produced in laboratory conditions that are reasonably analogous to the conditions of the exposure of atmospheric gases and surface ices in planetary settings in various natural environments, particularly in the outer Solar System. A number of tholins have been prepared in the context of the photochemical aerosols in Titan's atmosphere (e.g., Khare et al. 1984, Coll et al. 1999, Ramíriz et al. 2002, Tran et al. 2003, Imanaka et al. 2004), and while they are optically (spectrally) quite similar to Titan, they have until recently proven difficult to analyze and characterize fully from a chemical point of view. The recent work, for example by Tran et al. (2003), and Imanaka et al. (2004), has greatly improved the analysis and characterization of a class of tholins produced by photolysis and cold plasma irradiation of gaseous mixtures, as discussed below.

Optically, the tholins are characterized by strong absorption in the ultraviolet and visible spectral regions giving them strong yellow, orange, and red colors, high reflectance at longer wavelengths, and (in some cases) absorption bands characteristic of aliphatic and aromatic hydrocarbons with varying amounts of substituted nitrogen. We discuss this further in a later section.

\section{Models of Planetary Surfaces}

While simple spectral comparisons can be a guide to the presence or absence of tholins or other materials on a planetary surface or in an atmosphere, quantitative studies require the computation of the full spectral reflectance properties, which include not only the spectral shape, but the absolute reflectance (characterized by geometric albedo) across the full range of wavelengths for which observations exist. Such quantitative spectral matching is usually accomplished by the use of rigorous scattering models, of which the 
Hapke (1981, 1993) and Shkuratov et al. (1999) theories are often used. In addition to the original literature sources, the discussion of the practical application of Hapke theory to reflectance spectroscopy of solid surfaces by Verbiscer and Helfenstein (1998) is particularly useful. See also McEwen (1991). Poulet et al. (2002) have compared the Hapke and Shkuratov theories in the context of modeling solid surfaces, and Cruikshank et al. (2004) have described the computational procedures in more general terms.

Planetary surfaces and atmospheres are observed remotely both by the sunlight that is scattered from them and by their thermal emission arising from their natural temperatures. The specific wavelength regions of these two regimes depend on the characteristics of the individual Solar System body and on its distance from the Sun (hence its temperature). Typically, however, the scattered sunlight regime extends from the ultraviolet to about $5 \mu \mathrm{m}$ wavelength, and the thermal emission extends from about 5 $\mu \mathrm{m}$ to $200 \mu \mathrm{m}$. In this paper we are particularly interested in the spectral region where scattered sunlight dominates the flux detected from a Solar System body.

We note that the naturally occurring materials on a planetary surface can be segregated from one another or can be mixed in various combinations in a number of ways that affect the scattering properties and the abundances derived from the synthetic spectra calculated from scattering theories to match the observed spectra. These mixing scenarios have been discussed in various publications, including Poulet et al. (2002), Cruikshank et al. (1998), Roush and Cruikshank (2004), and Cruikshank et al. (2004).

\section{Tholins Used in Spectral Models}

Models using tholins as coloring agents successfully match the spectra of a number of both unusual and representative OSS bodies. Cruikshank et al. (1998b) matched the spectrum of the extremely red Centaur object 5145 Pholus with a model using the Khare et al. (1984) Titan tholin, $\mathrm{H}_{2} \mathrm{O}$ ice, $\mathrm{CH}_{3} \mathrm{OH}$ ice, and amorphous carbon; the Pholus spectrum has clear absorption bands in addition to the red color. Owen et al. (2001) calculated a simple model of the spectral reflectance of the low-albedo hemisphere of Saturn's satellite Iapetus $(0.4-3.8 \mu \mathrm{m})$ that successfully matches the red color and a strong absorption band $(2.7-3.6 \mu \mathrm{m})$ with the Triton tholin of McDonald et al. (1994) [the optical constants of Triton tholin, 0.2-10 $\mu \mathrm{m}$ are shown in Fig. 3 of Cruikshank et al. 2005]. Khare's Triton tholin was made by corona discharge in a gaseous mixture of $\mathrm{N}_{2}+\mathrm{CH}_{4}$ (1000:1) that approximately simulates the tenuous atmosphere of Neptune's satellite Triton. Dotto et al. (2004) matched the spectra of several Centaurs and Kuiper Belt objects having a wide range of color from nearly neutral to extremely red, also using the Titan tholin of Khare et al. (1984) in various combinations with other components $\left(\mathrm{H}_{2} \mathrm{O}\right.$ ice, amorphous carbon).

In a study of the rings of Saturn, Cuzzi and Estrada (1998) found that the particles in the $\mathrm{A}$ and $\mathrm{B}$ rings contain a material imparting a red color (absorption toward the violet spectral region), while the particles in the $C$ ring and Cassini's division are lower in albedo and less red in color. They note that, "No silicates have the appropriate combination of steep spectral slope and high absorptivity to explain the rings' visual 
color while remaining compatible with microwave observations." Titan tholin matches the colors and albedos of the particles when incorporated into the scattering models with $\mathrm{H}_{2} \mathrm{O}$ ice. To explain the lower albedo and more neutral color of the darker rings, Cuzzi and Estrada (1998) suggest that "material with properties like carbon black, as seen in at least some comets and interplanetary dust particles, is needed...". Subsequent modeling of the rings by Poulet and Cuzzi (2002) and Poulet et al. (2003) also incorporated tholins and amorphous carbon to achieve fits to the observational data $(0.3-4 \mu \mathrm{m})$.

\section{New Research on Tholins}

Tholins are refractory organic materials formed from reduced gas mixtures or ice mixtures with UV photolysis and/or charged particle irradiation (Sagan and Khare, 1979). In order to calculate scattering models of the spectra of OSS objects, the optical constants of tholins are required for comparison with the astronomical observations. One of the most successful measurements of the optical constants was done for Titan tholin by Khare et al. (1984). They produced Titan tholin from $\mathrm{CH}_{4} / \mathrm{N}_{2}=10 / 90$ gas mixture under DC plasma irradiation at $0.2 \mathrm{hPa}$ and measured the optical constants of Titan tholin from soft X-ray to microwave frequencies. The geometric albedo of Titan (its atmospheric haze) was explained fairly well using the derived optical constants of Titan tholin (McKay et al. 1989). Since then, some sets of optical constants (the complex refractive indices, $n$ and $k$ ) were measured for additional hydrocarbon tholins produced by charged particle irradiation of pure $\mathrm{CH}_{4}$ gas and $\mathrm{CH}_{4} / \mathrm{H}_{2}$ gas mixtures (Khare et al. 1987), for tholins produced by charged particle irradiation of $\mathrm{H}_{2} \mathrm{O} / \mathrm{C}_{2} \mathrm{H}_{6}=6 / 1$ ice mixtures at $77 \mathrm{~K}$ (Khare et al. 1993), for poly-HCN (Khare et al. 1994), and for kerogen and organic residue from the Murchison meteorite (Khare et al. 1990). Although those optical constants show wide variations, all of these organic materials show reddish colors with greater absorption at shorter wavelengths in the visible spectral region. [Note on pressure units: $1 \mathrm{hPa}=1$ hectoPascal $=10^{2}$ Pascal $=0.76$ torr $]$

In the early work the cause of the reddish color of tholins and the relationships between the optical proprieties and the chemical structure of tholins were not well understood. Although polycyclic aromatic hydrocarbons and some functional groups were suggested in Titan tholin (Khare et al., 1984; Sagan et al., 1993), other details of the chemical structure remain unclear. Many laboratory simulations have been conducted to investigate the optical and chemical properties of tholins (McDonald et al. 1994; McKay 1996; Coll et al. 1999, Ramirez et al. 2002). McDonald et al. (1994) compared the chemical properties of Titan tholin and Triton tholin that were produced from $\mathrm{CH}_{4} / \mathrm{N}_{2}=$ $10 / 90$ and $0.1 / 99.9$ gas mixtures respectively, and found distinct properties in these tholins with specific elemental compositions and chemical structures. McKay (1996) showed that optical properties vary with the tholins produced from different initial $\mathrm{CH}_{4} / \mathrm{N}_{2}$ ratios by comparing their transmittances in the ultraviolet-visible (UV/Vis) spectral ranges. Ramirez et al. (2002) measured the optical properties of tholin formed from a $\mathrm{CH}_{4} / \mathrm{N}_{2}$ gas mixture (2/98) at about $1 \mathrm{hPa}$, and found a ten times smaller value of $k$ (the imaginary component of the complex refractive index) in the UV/Vis ranges than those measured by Khare et al. (1984). This difference reveals that the properties of 
tholin may depend on the experimental conditions, such as initial ratio of $\mathrm{CH}_{4} / \mathrm{N}_{2}$ and deposition pressure.

Recently, Imanaka (2004) and Imanaka et al. (2004) systematically investigated a range of Titan tholins produced under various pressures in cold plasma. They found that the ratio of aromatic to aliphatic components monotonically decreased with increasing pressure and that the $\mathrm{k}$-value in the UV/Vis ranges correspondingly decreased by an order of magnitude. They suggested that the optical properties of tholin in the UV/Vis ranges may strongly depend on the delocalized electrons from aromatic components embedded in a saturated carbon matrix.

The absorption properties of tholin-like materials that show a reddish slope in the visible wavelength region merit further consideration. Tholins are a carbonaceous material containing $\mathrm{C}, \mathrm{H}, \mathrm{N}$, and $\mathrm{O}$, and while the exact structure is unknown, the fundamental building blocks are most likely composed of carbon, and probably nitrogen. Because of the different bond hybridization available $\left(\mathrm{sp}, \mathrm{sp}^{2}, \mathrm{sp}^{3}\right)$, carbonaceous materials show a wide variety of optical properties. For example, diamond is composed of tetrahedral $\mathrm{sp}^{3}$-hybridized C-C bonding configurations ( $\sigma$ bonds) and is transparent at visible wavelengths. Graphite is a trigonal $\mathrm{sp}^{2}$ network that forms planar six-fold rings. The completely delocalized $\pi$ electrons are responsible for the metal-like properties of graphite (its black color and absence of reddish color). In general, the absorption in the visible range depends on the $\square \square$ and $n-\square$ excitations of unsaturated bond structures. The more conjugation in a molecule, the more the absorption is displaced toward longer wavelength (e.g., Rao, 1975). Nitrogen atoms might help the delocalization because of the lone pair electrons. Tholin is a highly heterogeneous material containing both saturated and unsaturated bonds. Thus, the reddish slope of absorption in the visible range would depend on both abundance and size distributions of conjugated structures (either aromatics or alkenes). Since tholin is formed from simple molecules, the size distributions of conjugated structures would be controlled by its formation chemistry. Hydrogen is one of the main elements that break $\mathrm{C}=\mathrm{C}$ bonds, localizing the $\square$ electrons, and thus determines the size of delocalized $\square$ electrons. Thus, the role of hydrogen in the tholin formation (or modification) process would appear to be important in controlling the reddish absorption in UV/VIS wavelengths (Imanaka 2004).

In order to investigate Solar System bodies in the full range of wavelengths that are observed, it is important to obtain the optical constants of various tholins over a broad range of wavelengths in laboratory, including the near-infrared (NIR, 1-3 $\mu \mathrm{m})$ and midinfrared (MIR, $\sim 3-50 \mu \mathrm{m})$. In particular, the absorption properties of tholin in NIR range have not been well investigated because of the general weakness of any absorption in the continuum or in specific bands. Figure 1 shows the transmittance spectra of Titan tholins formed at five different pressures from $\mathrm{CH}_{4} / \mathrm{N}_{2}(=10 / 90)$ gas mixture by cold plasma irradiation, which were previously described in detail (Imanaka et al. 2004). The near infrared spectrum of Titan tholin formed at $0.26 \mathrm{hPa}$ is also shown. The sample thickness for NIR region is about twenty five times greater than those of other samples in MIR region, so that the absorption peak intensities in NIR are much smaller than in MIR. Several overtones of $\mathrm{C}-\mathrm{H}, \mathrm{N}-\mathrm{H}$, and $\mathrm{C} \square \mathrm{N}$ stretching bands and combination bands are 
preliminarily identified in the NIR range. The reddish absorption slope extends from the visible range to about $1.8 \mu \mathrm{m}$. It would be important to compare simultaneously the reddish slope in the UV/VIS ranges and absorption bands in NIR/MIR ranges using the optical properties of various tholins with observations of OSS objects over a wide range of wavelength.

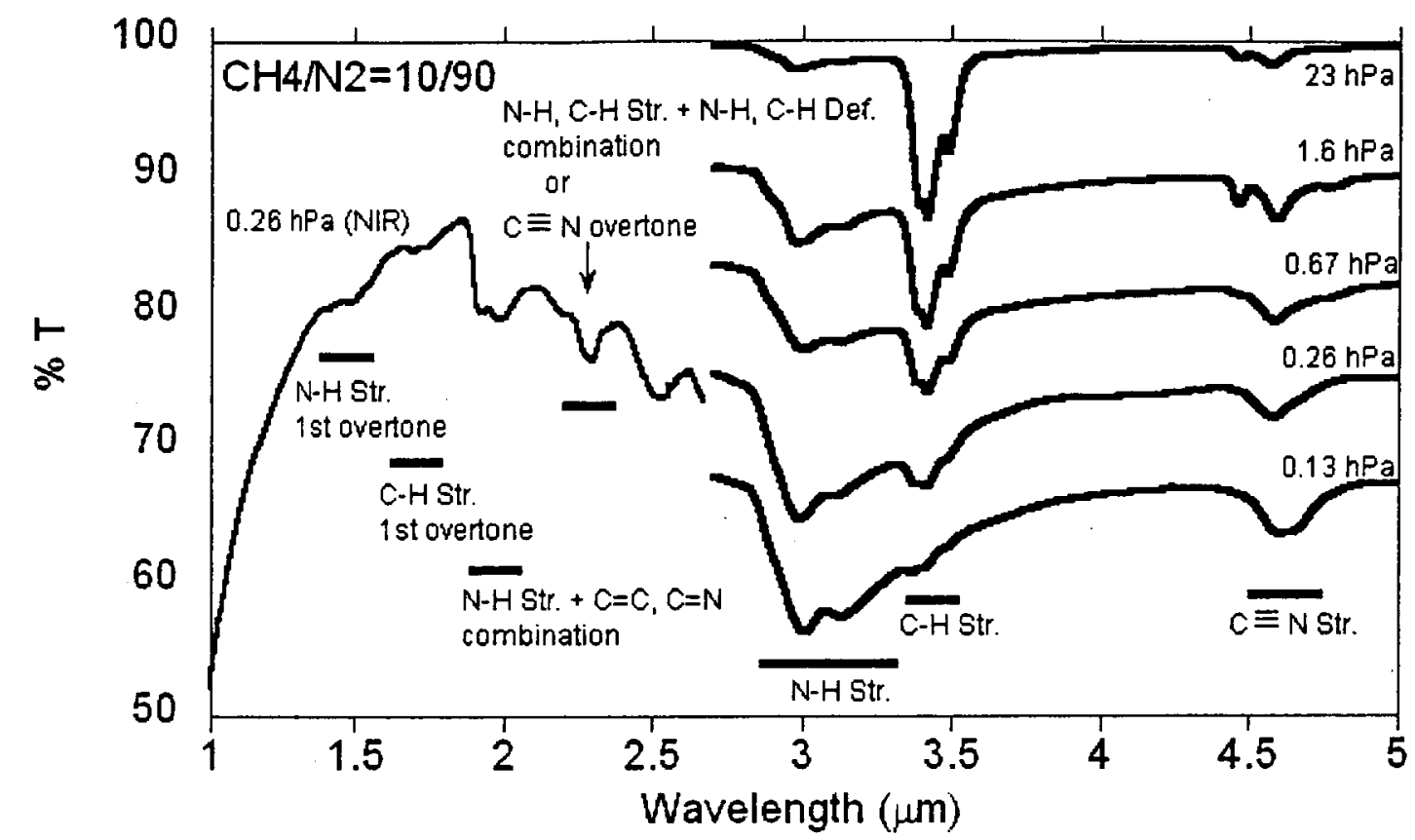

Figure 1. Transmission spectra of Titan tholins formed by cold plasma discharge in a $\mathrm{CH}_{4} / \mathrm{N}_{2}=10 / 90$ gas mixture at five different pressures. The spectrà have been offset from one another for clarity. The NIR spectra of Titan tholin formed at $0.26 \mathrm{hPa}$ was obtained from a sample about twenty five times thicker than the others, so that the absorption peak intensities in the NIR are much smaller than in the MIR. The reddish absorption slope extends from the visible range to about $1.8 \mu \mathrm{m}$.

In a systematic study of the methane-nitrogen tholins, Imanaka (2004) has also investigated the variation in spectral properties of tholins made at a uniform pressure and temperature but with variable amounts of $\mathrm{CH}_{4}$ in the mixture. Figure 2 shows transmittance spectra of tholins formed at $1.6 \mathrm{hPa}$, but from different ratios of $\mathrm{CH}_{4} / \mathrm{N}_{2}$ in the initial gas mixtures. The ratios of the areas of the aliphatic $\mathrm{C}-\mathrm{H}$ bands $(\sim 3.4 \mu \mathrm{m})$ to $\mathrm{N}-\mathrm{H}$ bands $(\sim 2.8-3.4 \mu \mathrm{m})$ increase monotonically, but only slightly, with the increase of $\mathrm{CH}_{4}$ ratio in the initial $\mathrm{CH}_{4} / \mathrm{N}_{2}$ gas mixture. This variation in the ratio of $\mathrm{C}-\mathrm{H}$ bands to $\mathrm{N}$ $\mathrm{H}$ bands is smaller than that in tholins formed at different pressures (see Fig. 1). The absorption properties in the UV/Vis ranges do not change significantly, except for the pure $\mathrm{CH}_{4}$ tholin. Thus, the optical properties of tholin formed from $\mathrm{CH}_{4} / \mathrm{N}_{2}$ gas mixtures are dominated by the deposition pressures rather than their initial mixing ratio used in this study. 


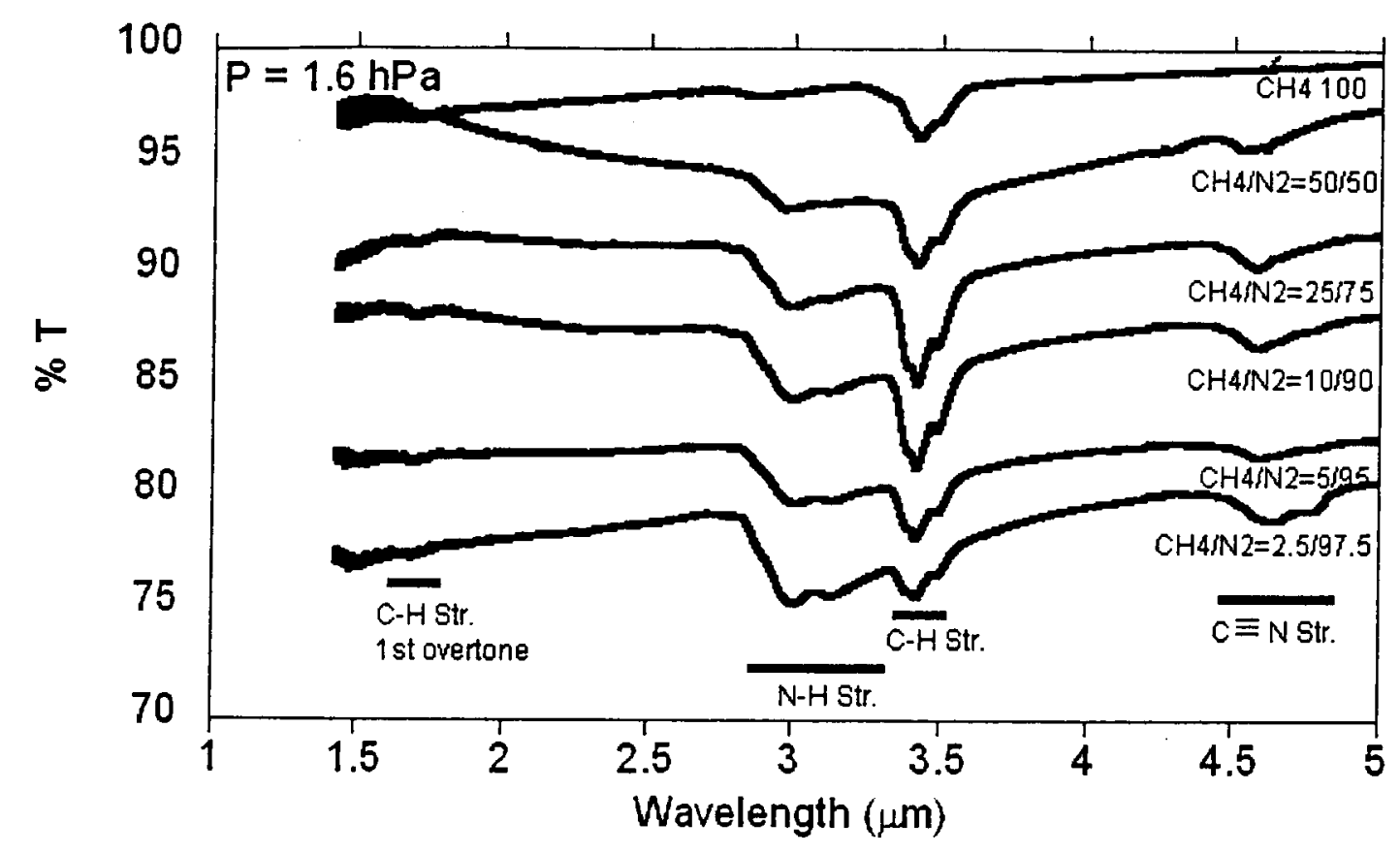

Figure 2. Transmission spectra of tholins formed by cold plasma discharge at $1.6 \mathrm{hPa}$ from $\mathrm{CH}_{4} / \mathrm{N}_{2}$ gas mixtures with various initial ratios. Intensities have been offset. The absorption intensity of $\mathrm{N}-\mathrm{H}$ bands around $3.2 \mu \mathrm{m}$ increases monastically when the $\mathrm{CH}_{4}$ ratio in initial gas mixture decreases. The absorption intensity of $\mathrm{C}-\mathrm{H}$ bands around 3.4 $\mu \mathrm{m}$ has a maximum when the $\mathrm{CH}_{4}$ ratio is $\sim 10-25 \%$ in the initial gas mixture.

Additional properties of the Titan tholins produced in this investigation were discussed in greater detail in Imanaka (2004) and Imanaka et al. (2004), and are briefly summarized here:

1) Both the quantity and size of aromatic ring compounds in tholin increase with decreasing deposition pressure.

2) The abundance of saturated $\mathrm{C}-\mathrm{H}$ bonds is lower in tholins formed at low pressures, while $\mathrm{N}-\mathrm{H}$ bonds become more abundant.

3) More nitrogen is included in carbon networks at lower pressures in structures such as carbon diimides $(-\mathrm{N}=\mathrm{C}=\mathrm{N}-)$ and isocyanides $(-\mathrm{C}-\mathrm{N} \square \mathrm{C})$, while at higher deposition pressures nitrogen exists as a terminal group, such as nitrile $(-\mathrm{C} \square \mathrm{N})$.

4) Tholins formed at low pressures contain clusters of $\mathrm{N}$-containing polycyclic aromatic compounds in a matrix of $\mathrm{C}$ and $\mathrm{N}$ branched chain networks which are connected tightly to one another with hydrogen bonding of $\mathrm{N}-\mathrm{H}$ bonds. Tholin formed at high pressure (23 $\mathrm{hPa}$ ) consists of polymer-like branched chain structures terminated with $-\mathrm{CH}_{3},-\mathrm{NH}_{2}$, and $-\mathrm{C} \square \mathrm{N}$ with fewer aromatic compounds.

5) $\mathrm{The} \mathrm{C} / \mathrm{N}$ ratio in tholins increases from a value between 1.5 and 2 at lower pressures to 3 at high pressure $(23 \mathrm{hPa})$. Thus, the $\mathrm{N}$ is incorporated into tholin more efficiently at low pressures.

6) Reddish-brown tholin films formed at low pressures $(0.13$ and $0.26 \mathrm{hPa})$ show stronger UV/Vis absorptions than yellowish tholin films formed at higher pressures (1.6 
and $23 \mathrm{hPa}$ ). The imaginary part of the complex refractive indices at UV-Vis wavelengths depends strongly on the delocalized $\square$ electrons from aromatic and N-containing, heteroaromatic compounds in tholins.

\section{Conclusion}

Together with ices and minerals, organic solid material is emerging as a significant component of the suite of constituent materials of small Solar System bodies. In this paper we have summarized some of the astronomical evidence, particularly in terms of the colors that even small concentrations of complex organic solids impart to the surface of an object. We have focused on recent laboratory work on the synthesis and analysis of a tholin formed by energetic processing of a gas mixture chosen to simulate the composition of Titan's atmosphere, at a number of different pressures chosen to simulate different pressure levels (hence altitudes) in that atmosphere. This systematic study, reported in detail by Imanaka (2004) and Imanaka et al. (2004) has begun to elucidate the details of several aspects of the formation pathways and the chemical structure of Titan tholin. Energetic processing of other gas mixtures and of condensed ices also produces complex refractory organic solids of probable relevance to outer Solar System bodies, and while preliminary experiments with these materials have produced promising results, much more work is needed.

\section{Acknowledgments}

Imanaka acknowledges support from NASA's Exobiology program under grants NCC2-1118, and NCC2-1358, as well as support from the Japanese Scientific Foundation. Cruikshank and Dalle Ore are supported in part by NASA's Cassini mission and the Spitzer Science Center, and previously by NASA's Planetary Astronomy program.

\section{References}

Binder, A. B., and Cruikshank, D. P. and mineralogical investigation of the surface of Mars. Icarus 5, 521-525, 1966.

Coll, P., Coscia, D., Smith, N., et al. Experimental laboratory simulation of Titan's atmosphere (aerosols and gas phase). Planet. Space Sci. 47, 1331-1340, 1999.

Cruikshank, D. P., Roush, T. L., Owen, et al. The surface compositions of Triton, Pluto, and Charon, in: Schmitt, B., Festou, M., and de Bergh, C., (Eds.) Solar System Ices, Kluwer, pp. 579-606, 1998a.

Cruikshank, D. P. Roush, T. L., Bartholomew, M. J., et al. The composition of Centaur 5145 Pholus. Icarus 135, 389-407, 1998 b. 
Cruikshank, D. P., Dalle Ore, C. M., Roush, T. L., et al. Constraints on the composition of Trojan asteroid 624 Hektor. Icarus 153, 348-360, 2001.

Cruikshank, D. P., Roush, T. L., and Poulet, F. Quantitative modeling of the spectral reflectance of Kuiper Belt objects and Centaurs. C. R. Physique 4, 783-789, 2004.

Cruikshank, D. P., Owen, T. C., Dalle Ore, C., M., et al. A spectroscopic study of the surfaces of Saturn's large satellites: $\mathrm{H}_{2} \mathrm{O}$ ice, tholins, and minor constituents. Icarus (in press) 2005.

Cuzzi, J. N., and Estrada, P. R. Compositional evolution of Saturn's rings due to meteoroid bombardment. Icarus 132, 1-35, 1998.

Dotto, E., Barucci, M. A., and de Bergh, C. Surface composition of TNOs and Centaurs: visible and near-infrared spectroscopy. C. R. Physique 4, 775-782, 2004.

Gradie, J. and Veverka, J. The composition of the Trojan asteroids. Nature 283, 840$842,1980$.

Hapke, B. Bidirectional reflectance spectroscopy. I. Theory. J. Geophys. Res. 96, 3039-3054, 1981.

Hapke, B. Theory of Reflectance and Emittance Spectroscopy. Cambridge Univ. Press, New York. 455 pp., 1993.

Hapke, B. Space weathering. J. Geophys. Res. 106, 10,039-10,073, 2001.

Emery, J. P., and Brown, R. H. The surface composition of Trojan asteroids: Constraints set by scattering theory. Icarus 170, 131-152, 2004.

Imanaka, H. Laboratory Simulations of Titan's Organic Haze and Condensation Clouds, Ph. D. thesis, Univ. of Tokyo, Tokyo, 2004.

Imanaka, H., Khare, B. N., Elsila, J. E., et al. Laboratory experiments on Titan tholin formed in cold plasma at various pressures: Implications for nitrogen-containing polycyclic aromatic compounds in Titan haze. Icarus 168, 344-366, 2004.

Kerridge, J., Chang, S., and Shipp, R. Isotopic characterization of kerogen-like material in the Murchison carbonaceous chondrite. Geochim. Cosmochim. Acta 51, 2527-2540, 1987.

Khare, B. N., Sagan, C., Arakawa, E. T., et al. Optical constants of organic tholins produced in a simulated Titanian atmosphere: From soft $\mathrm{x}$-ray to microwave frequencies. Icarus 60, 127-137, 1984. 
Khare, B.N., Sagan, C., Thompson, W. R. Solid Hydrocarbon Aerosols Produced in Simulated Uranian and Neptunian Stratospheres, J. Geophys. Res. 92, 15067-15082, 1987.

Khare, B.N., Thompson, W.R., Sagan, C., et al. Optical constants of kerogen from 0.15 to $40 \mu \mathrm{m}$ : Comparison with meteoritic organics, in: Proc. Int. Conf. Lab. Res. Planetary Atmospheres, $1^{\text {st }}$, NASA CP-3077, 340 - 356, 1990.

Khare, B.N., Thompson, W. R., Cheng, L. et al. Production and Optical Constants of Ice Tholin from Charged Particle Irradiation of $(1: 6) \mathrm{C}_{2} \mathrm{H}_{6} / \mathrm{H}_{2} \mathrm{O}$ at $77 \mathrm{~K}$, Icarus 103, 290 300, 1993.

Khare, B.N., Sagan, C., Thompson, W. R., et al. Optical properties of poly-HCN and their astronomical applications, Can. J. Chem. 72, 678 - 694, 1994.

McDonald, G. D., Thompson, W. R., Heinrich, M., et al. Chemical investigation of Titan and Triton tholins. Icarus 108, 137-145, 1994.

McEwen, A. S. Photometric functions for photoclinometry and other applications. Icarus 92, 298-311, 1991.

McKay, C.P., Pollack, J. B., and Courtin, R. The Thermal Structure of Titan's Atmosphere, Icarus 80, 23-53, 1989.

McKay, C.P., Elemental composition, solubility, and optical properties of Titan's organic haze, Planet. Space Sci. 44, 741-747, 1996.

Owen, T. C., Cruikshank, D. P., Dalle Ore, C. M., et al. Decoding the Domino: The Dark Side of Iapetus. Icarus 149, 160-172, 2001.

Pendleton, Y. J., and Allamandola, L. J. The organic refractory material in the diffuse interstellar medium: Mid-infrared spectroscopic constraints. Astrophys. J. Supp. 138, 75-98, 2002.

Pieters, C. M., Taylor, L. A., Noble, S. K., et al. Space weathering on airless bodies: Resolving a mystery with lunar samples. Meteoritics and Planetary Sci. 35, 1101-1107, 2000.

Poulet, F., and Cuzzi, J. N. The composition of Saturn's rings. Icarus 160, 350-358, 2002.

Poulet, F., Cuzzi, J. N., Cruikshank, D. P., et al. Comparison between the Shkuratov and Hapke scattering theories for solid planetary surfaces. Application to the surface composition of two Centaurs. Icarus 160, 313-324, 2002. 
Poulet, F., Cruikshank, D. P., Cuzzi, J. N., et al. Compositions of Satum's rings A, B, and $\mathrm{C}$ from high spectral resolution near-infrared spectroscopic observations. Astron. Astrophys. 412, 305-316, 2003.

Ramírez, S. I., Coll, P., da Silva, A., et al. Complex refractive index of Titan's aerosol analogues in the 200-900 nm domain. Icarus 156, 515-529, 2002.

Rao, C.N.R., Ultra-Violet and Visible Spectroscopy. Chemical Applications, 242 pp., Butterworth, London, 1975.

Roush, T. L., and Cruikshank, D. P. Observations and laboratory data of planetary organics, in: Ehrenfruend, P. et al. (Eds.), Astrobiology: Future Perspectives. Kluwer Academic Publishers, Dordrecht. 149-177, 2004.

Sagan, C., and Khare, B. N. Tholins: organic chemistry of interstellar grains and gas, Nature 277, 102-107, 1979.

Sagan, C., Khare, B., N., Thompson, W. R. et al. Polycyclic Aromatic Hydrocarbons in the Atmosphere of Titan and Jupiter, Astrophys. J. 414, 399 - 405, 1993.

Shkuratov, Y., Starukhina, L., Hoffmann, H., and Arnold, G. A model of spectral albedo of particulate surfaces. Implications for optical properties of the Moon. Icarus 137, 235246, 1999.

Tran, B. N., Ferris, J. P., and Chera, J. J. The photochemical formation of a Titan haze analog. Structural analysis by X-ray photoelectron and infrared spectroscopy. Icarus 162, 114-124, 2003.

Verbiscer, A., and Helfenstein, P. 1998. Reflectance spectroscopy of icy surfaces, in: Schmitt, B., Festou, M., and de Bergh, C., (Eds.) Solar System Ices. Kluwer, pp. 157197. 


\section{Figure Captions:}

\section{Figure 1.}

Transmission spectra of Titan tholins formed by cold plasma discharge in a $\mathrm{CH}_{4} / \mathrm{N}_{2}=$ 10/90 gas mixture at five different pressures. The spectra have been offset from one another for clarity. The NIR spectra of Titan tholin formed at $0.26 \mathrm{hPa}$ was obtained from a sample about twenty five times thicker than the others, so that the absorption peak intensities in the NIR are much smaller than in the MIR. The reddish absorption slope extends from the visible range to about $2 \mu \mathrm{m}$.

\section{Figure 2.}

Transmission spectra of tholins formed by cold plasma discharge at $1.6 \mathrm{hPa}$ from $\mathrm{CH}_{4} / \mathrm{N}_{2}$ gas mixtures with various initial ratios. Intensities have been offset. The absorption intensity of $\mathrm{N}-\mathrm{H}$ bands around $3.2 \mu \mathrm{m}$ increases monotonically when the $\mathrm{CH}_{4}$ ratio in initial gas mixture decreases. The absorption intensity of $\mathrm{C}-\mathrm{H}$ bands around $3.4 \mu \mathrm{m}$ has a maximum when the $\mathrm{CH}_{4}$ ratio is $\sim 10-25 \%$ in the initial gas mixture. 